\title{
6-(7-Nitro-2,1,3-benzoxadiazol-4-ylthio)hexanol, a specific glutathione $S$-transferase inhibitor, overcomes the multidrug resistance (MDR)-associated protein 1-mediated MDR in small cell lung cancer
}

\author{
Giuseppe Filomeni, ${ }^{1}$ Paola Turella, ${ }^{2}$ \\ Maria Luisa Dupuis, ${ }^{3}$ Olindo Forini, ${ }^{4}$ \\ Maria Rosa Ciriolo, ${ }^{1,5}$ Maurizio Cianfriglia, ${ }^{3}$ \\ Silvia Pezzola, ${ }^{2}$ Giorgio Federici, ${ }^{6}$ \\ and Anna Maria Caccuri ${ }^{2}$ \\ Departments of ${ }^{1}$ Biology, and ${ }^{2}$ Chemical Sciences and \\ Technologies, University of Rome "Tor Vergata"; ${ }^{3}$ Drug Research \\ and Evaluation, "Istituto Superiore di Sanità"; ${ }^{4}$ Internal Medicine, \\ University of Rome "Tor Vergata"; 5 Research Centre, Istituto \\ di Ricovero e Cura a Carattere Scientifico San Raffaele Pisana; \\ and ${ }^{6}$ Children's Hospital, Istituto di Ricovero e Cura a Carattere \\ Scientifico "Bambin Gesu," Rome, Italy
}

\begin{abstract}
In the present work, we have investigated the antitumor activity of 6-(7-nitro-2,1,3-benzoxadiazol-4-ylthio)hexanol (NBDHEX) on aggressive small cell lung cancer. NBDHEX not only is cytotoxic toward the parental small cell lung cancer $\mathrm{H} 69$ cell line $\left(\mathrm{LC}_{50}\right.$ of $\left.2.3 \pm 0.6 \mu \mathrm{mol} / \mathrm{L}\right)$ but also overcomes the multidrug resistance of its variant, H69AR, which overexpresses the ATP-binding cassette transporter multidrug resistance - associated protein 1 (MRP1; LC $_{50}$ of $4.5 \pm 0.9 \mu \mathrm{mol} / \mathrm{L}$ ). Drug efflux experiments, done in the presence of a specific inhibitor of MRP1, confirmed that NBDHEX is not a substrate for this export pump. Interestingly, NBDHEX triggers two different types of cell death: a caspase-dependent apoptosis in the H69AR cells and a necrotic phenotype in the parental $\mathrm{H} 69$ cells. The apoptotic pathway triggered by NBDHEX in H69AR cells is associated with c-Jun $\mathbf{N H}_{2}$-terminal kinase and c-Jun activation, whereas glutathione oxidation and activation of $\mathrm{p} 38^{\mathrm{MAPK}}$ is observed in the NBDHEX-treated $\mathrm{H} 69$ cells. In contrast to the parental cells, the higher propensity to die through apoptosis of the H69AR cell line may be related to the lower expression of the antiapoptotic protein
\end{abstract}

\footnotetext{
Received 7/24/07; revised 11/27/07; accepted 12/20/07.

Grant support: Istituto Superiore di Sanità-NIH research grant.

The costs of publication of this article were defrayed in part by the payment of page charges. This article must therefore be hereby marked advertisement in accordance with 18 U.S.C. Section 1734 solely to indicate this fact.

Note: G. Filomeni and P. Turella contributed equally to this work.

Requests for reprints: Anna Maria Caccuri, Department of Chemical Sciences and Technologies, University of Rome "Tor Vergata," Via della Ricerca Scientifica, 00133 Rome, Italy. Phone: 39-0672594378; Fax: 39-0672594328. E-mail: caccuri@uniroma2.it

Copyright $@ 2008$ American Association for Cancer Research. doi:10.1158/1535-7163.MCT-07-0487
}

Bcl-2. Therefore, down-regulation of a factor crucial for cell survival makes H69AR cells more sensitive to the cytotoxic action of NBDHEX, which is not a MRP1 substrate. We have previously shown that NBDHEX is cytotoxic toward P-glycoprotein-overexpressing tumor cell lines. Therefore, NBDHEX seems a very promising compound in the search for new molecules able to overcome the ATP-binding cassette family of proteins, one of the major mechanisms of multidrug resistance in cancer cells. [Mol Cancer Ther 2008;7(2):371 -9]

\section{Introduction}

Lung cancer is the leading cause of cancer-related deaths and is classified clinically into two major histologic types: small cell lung cancer (SCLC), which accounts for $\sim 25 \%$ of cases, and non-SCLC, which accounts for the remaining $75 \%$. SCLC is a particularly aggressive form of lung cancer and has an extremely poor prognosis $(1,2)$. Current information suggests that, in most cells, defects in apoptosis signaling are the most critical determinants for tumor development and progression $(3,4)$. The antiapoptotic protein Bcl-2 is a critical survival factor for SCLC cells, and it has been shown that down-regulation of $\mathrm{Bcl}-2$ induces apoptosis and enhances chemosensitivity in these tumor cells $(5,6)$. Moreover, after an initial response to chemotherapy, there is a relapse in most patients with SCLC, and their tumors become largely refractory to further treatments. P-glycoprotein (P-gp) and the multidrug resistance (MDR)-associated protein 1 (MRP1) are the most extensively studied ATP-binding cassette transporters and are often regarded as the prototypes for understanding the cell-based mechanisms of MDR (7-9). The clinical importance of P-gp and MRP1 stems from the fact that, in parallel with their overexpression, the tumor cells simultaneously exhibit acquired cross-resistance to diverse chemotherapeutic agents, resulting in the failure of chemotherapy for many cancers. To study the MDR phenotype of SCLC, Mirsk et al. (10) have developed a drug-resistant SCLC cell line by selection with Adriamycin. This cell line, designated H69AR, does not overexpress P-gp. Thus, it provides a model system for investigating MRP1-mediated MDR. We have recently reported that 6-(7-nitro-2,1,3-benzoxadiazol-4-ylthio)hexanol (NBDHEX) is not a substrate of the P-gp export pump and both P-gpoverexpressing tumor cells and their parental cell lines are efficiently committed to death by this molecule (11). NBDHEX is a nonpolar compound that behaves as a suicide inhibitor for glutathione $S$-transferases (GST). In fact, it is conjugated with reduced glutathione (GSH), so 
leading to a stable $\sigma$ complex in the GST active site (12). The MRP1 export pump is able to transport hydrophobic anions, including drugs conjugated with GSH (GS-X pump), glucuronide, and sulfate. In addition, the MRP1 pump removes unmodified anticancer agents cotransported with GSH $(13,14)$. Therefore, the anionic adduct between NBDHEX and GSH could represent a substrate for the MRP1 transporter and it may be extruded from the cell. In the present work, we have analyzed the activity of NBDHEX against both H69 tumor cells and their Adriamycin-resistant counterpart H69AR, which overexpresses MRP1.

\section{Materials and Methods}

Cell Lines and MDR Typing with Monoclonal Antibodies

Human SCLC H69 and its Adriamycin-selected, multidrug-resistant H69AR cell variant were kindly provided by Dr. Susan P. Cole (Department of Pathology, Queen's University, Kingston, Ontario, Canada). Cells were grown in RPMI 1640 supplemented with 10\% FCS and antibiotics. H69AR cells were maintained by alternate feedings with drug-free medium or medium containing $1.7 \mu \mathrm{mol} / \mathrm{L}$ Adriamycin. The stability of the MDR phenotype was periodically assessed by testing the MRP1 expression with the MRP1-specific monoclonal antibody MRPm6 (VinciBiochem; ref. 15). MDR cells were also typed for possible P-gp expression with the monoclonal antibody MM4.17, which recognizes an extracellular P-gp epitope (16).

\section{Cell Viability Test}

Cell viability testing was done using the sulforhodamine B assay (17). In brief, H69 and H69AR cells were placed in 96-well microtiter plates at a density of $0.7 \times 10^{6} / \mathrm{mL}$ and then incubated $48 \mathrm{~h}$ with NBDHEX at the desired final concentration (from 0.05 to $20 \mu \mathrm{mol} / \mathrm{L}$ ). A positive control was also done by treating both $\mathrm{H} 69$ and H69AR cells with $10 \mu \mathrm{mol} / \mathrm{L}$ puromycin, a dose that causes $100 \%$ cell death after $48 \mathrm{~h}$ (data not shown). After $48 \mathrm{~h}$ of incubation, cell growth was evaluated by an in situ cell fixation procedure followed by sulforhodamine B staining of proteins. In order not to exceed the absorbance linearity range, a suboptimal wavelength $(490 \mathrm{~nm})$ was used to read the samples (17). The absorbance value of the positive control was subtracted from that of both NBDHEX-treated and untreated control samples. The dose-response profile obtained fulfills the $\mathrm{LC}_{50}$ for NBDHEX (the concentration used to obtain $50 \%$ cellular mortality).

\section{Cell Treatments}

Treatments were done on cells cultured at $0.8 \times 10^{6} / \mathrm{mL}$. NBDHEX was synthesized as reported by Ricci et al. (12). Stock solutions of this compound were prepared in DMSO and diluted to the appropriate concentration in RPMI 1640 cell medium just before use.

The caspase-dependent nature of the NBDHEX-induced apoptosis was confirmed by measuring caspase activity and apoptosis in cells treated with the general caspase inhibitor Z-VAD-fmk at a concentration of $40 \mu \mathrm{mol} / \mathrm{L}$. The inhibitor was added to the cell culture $1 \mathrm{~h}$ before the addition of $3 \mu \mathrm{mol} / \mathrm{L}$ NBDHEX and maintained throughout the experimental time.

The protective effect of GSH on the NBDHEX-induced necrosis (H69) and apoptosis (H69AR) was determined after the addition to the cells of $1 \mathrm{mmol} / \mathrm{L}$ glutathione ethylester $1 \mathrm{~h}$ before the addition of $5 \mu \mathrm{mol} / \mathrm{L}$ NBDHEX.

Flow Cytometric Detection of Functional Drug Efflux

Cells were incubated for $1 \mathrm{~h}$ in the presence of $1.7 \mu \mathrm{mol} / \mathrm{L}$ Adriamycin or $10 \mu \mathrm{mol} / \mathrm{L}$ NBDHEX in the presence or absence of $50 \mu \mathrm{mol} / \mathrm{L}$ MK571 (VinciBiochem), a specific inhibitor of MRP1 (18). Then, cells were incubated for $1 \mathrm{~h}$ in drug-free medium with or without MK571 to give the opportunity for efflux or efflux blocking to occur. Efflux was stopped by pelleting the cells and adding ice-cold medium. Fluorescence of Adriamycin or NBDHEX was analyzed by a FACSCalibur flow cytometer (BD Biosciences) equipped with an argon laser source.

\section{Determination of Apoptosis and Necrosis}

Percentages of apoptosis and necrosis were determined on cells treated for $24 \mathrm{~h}$ with different concentrations of NBDHEX (from 0 to $5 \mu \mathrm{mol} / \mathrm{L}$ ).

For apoptosis determination, aliquots of cells were stained for 10 min with Hoechst 33342 and examined by fluorescence microscopy. For necrosis determination, aliquots of cells were stained with trypan blue and examined by microscopy using a Neubauer hemacytometer. Alternatively, cells were stained with $50 \mu \mathrm{g} / \mathrm{mL}$ propidium iodide, permeabilized with NP40 $(0.1 \%, \mathrm{w} / \mathrm{v})$, and analyzed by a FACSCalibur instrument.

\section{Glutathione Determination}

The intracellular levels of both reduced (GSH) and oxidized form (GSSG) of glutathione were determined at different times from the treatment with $3 \mu \mathrm{mol} / \mathrm{L}$ NBDHEX, as previously reported (19). Data are expressed as nmol of GSH or GSSG equivalents/mg of protein.

\section{Enzyme Activity}

GST and caspase-3 activities were measured as described previously (20). Protein concentration was determined by the bicinchoninic acid protein assay reagent (Pierce).

\section{Western Blotting Analysis}

Immunoblotting analysis was done on H69 and H69AR cell lines. At each time point analyzed, pellets of untreated control cells and cells treated with $3 \mu \mathrm{mol} / \mathrm{L}$ NBDHEX were lysed for Western blot analyses as described previously (20). Twenty micrograms of proteins were loaded on $12 \%$ polyacrylamide gel and transferred onto a nitrocellulose membrane (Bio-Rad). Monoclonal antiphosphorylated c-Jun $\mathrm{NH}_{2}$-terminal kinase (JNK) and anti-phosphorylated c-Jun (1:500; Santa Cruz Biotechnology); polyclonal anti-JNK and anti-c-Jun (1:1,000; Upstate Biotechnology), anti-p38 ${ }^{\text {MAPK }}$ (1:1,000; Santa Cruz Biotechnology), anti-phosphorylated isoform of p38 ${ }^{\mathrm{MAPK}}(1: 1,000$; Cell Signaling Technology and New England Biolabs), anti- $\beta$-actin (1:5,000; Sigma), and anti- $\alpha$-tubulin; and monoclonal anti-Bcl-2 (1:500; Dako) and anti-GSTP1-1 (1:2,000; Calbiochem) were used as primary antibodies. The protein complex formed on incubation with specific 
A

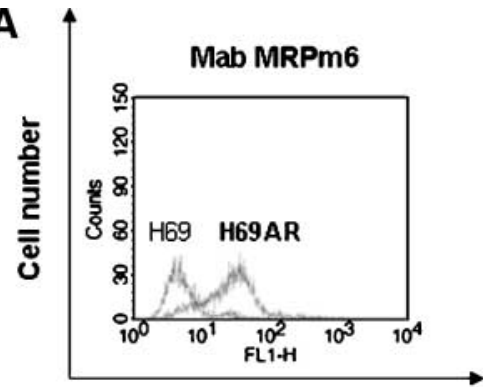

Fluorescence intensity

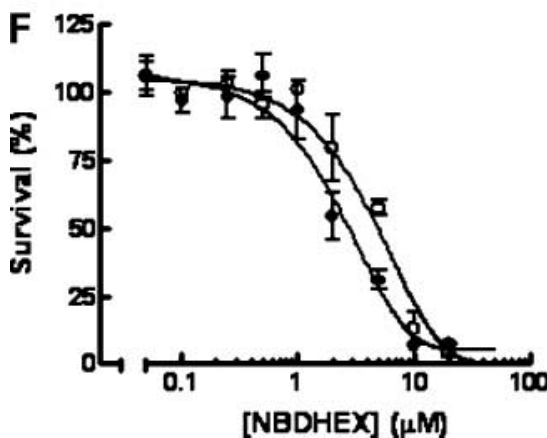

B

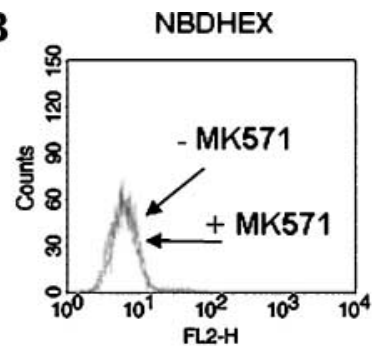

H69AR

D

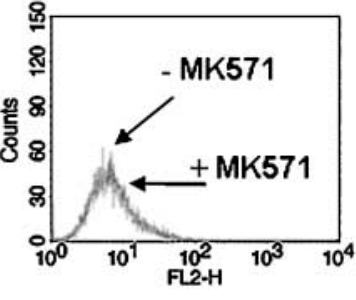

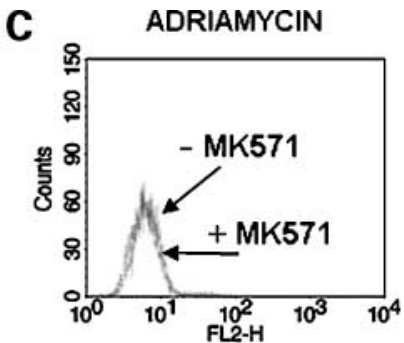

E

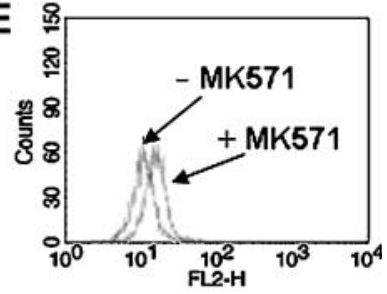

Figure 1. NBDHEX is not a substrate for MRP1 export pump and shows cytotoxic activity toward both H69 and H69AR cell lines. A, MRP1 expression was evaluated by flow cytometric analyses of $\mathrm{H} 69$ and H69AR cells incubated with monoclonal antibody (Mab) MRPm6. The effect of 50 mmol/L MRP1 inhibitor MK571 was determined on both H69 and H69AR cell lines. Cells were treated with either $10 \mu \mathrm{mol} / \mathrm{L}$ NBDHEX or $1.7 \mu \mathrm{mol} / \mathrm{L}$ Adriamycin alone or in combination with MK571. B, H69 with NBDHEX and with NBDHEX and MK571. C, H69 with Adriamycin and with Adriamycin and MK571. D, H69AR with NBDHEX and with NBDHEX and MK571. E, H69AR with Adriamycin and with Adriamycin and MK571. F, cell survival at different NBDHEX concentrations (from 0.05 to $20 \mu \mathrm{mol} / \mathrm{L}$ ) was determined by sulforhodamine B assay after $48 \mathrm{~h}$ of treatment. The dose-response profile for NBDHEX yields $\mathrm{LC}_{50}$ s of $2.3 \pm 0.6 \mu \mathrm{mol} / \mathrm{L}$ with $\mathrm{H} 69$ cells $(\bullet)$ and $4.5 \pm 0.9 \mu \mathrm{mol} / \mathrm{L}$ with $\mathrm{H} 69 \mathrm{AR}$ cell line $(0 ; P<0.05)$. Points, mean of three independent experiments, each done in quadruplicate; bars, SD.

secondary antibodies (1:10,000; Bio-Rad) was identified using FluorChem Imaging System (Alpha Innotech) after incubation with ChemiGlow chemiluminescence substrate (Alpha Innotech).

Densitometric analyses of proteins were done using the software Quantity One (Bio-Rad), and protein levels were normalized to the density of related $\beta$-actin bands.

\section{Data Presentation}

All of the experiments were repeated at least thrice. Results are presented as mean \pm SD. Statistical evaluation was done, where appropriate, using the Student's $t$ test or ANOVA followed by correction with Bonferroni's $t$ test. The criterion for statistical significance used was $P<0.05$.

\section{Results}

\section{NBDHEX Is Not a Substrate of MRP1}

Flow cytometry studies, conducted by specific antibodies, show that MRP1 is not present in the drug-sensitive H69 parental cell line. On the other hand, this export pump is highly expressed in the MDR cell variant H69AR, as assessed by the intracellular detection of the MRPm6 epitope by its cognate monoclonal antibody (Fig. 1A). In contrast, P-gp was not detected in both cell lines by the anti-P-gp monoclonal antibody MM4.17 (data not shown). Thus, the H69 and H69AR drug-sensitive/resistant cell pair provides a model system for investigating MRP1-mediated
MDR. To clarify if NBDHEX efflux could be mediated by MRP1, we did experiments in the presence of MK571. This molecule is known to specifically inhibit the MRP1mediated transport, so inducing intracellular accumulation of MRP1 substrates such as Adriamycin. As expected, MK571 inhibited Adriamycin efflux in H69AR cells (Fig. 1E), although it did not affect the intracellular concentration of Adriamycin in the sensitive variant (Fig. 1C). Conversely, MK571 did not affect the intracellular concentration of NBDHEX in both sensitive and resistant cell lines (Fig. 1B and D, respectively).

Moreover, a recently isolated NBDHEX-resistant cell variant of the human T-lymphoblastic leukemia CCRFCEM does not show detectable MRP1 or P-gp multidrug transporters (data not shown). ${ }^{7}$ These findings confirm the fact that NBDHEX is not a substrate for these export pumps.

\section{Cytotoxic Effect of NBDHEX on SCLC Cell Lines}

The ability of NBDHEX to cause cell death in H69 and H69AR cells is affected by an increase of cell density. Thus, taking advantage of the wide range of linearity of the sulforhodamine B assay, the NBDHEX cytotoxic activity

\footnotetext{
${ }^{7}$ M.L. Dupuis, in preparation.
} 
has been determined by using a cell density comparable with that used in the apoptosis/necrosis experiments. Under these experimental conditions, the dose-response profiles revealed a good cytotoxic activity both in sensitive H69 cell line ( $\mathrm{LC}_{50}$ of $\left.2.3 \pm 0.6 \mu \mathrm{mol} / \mathrm{L}\right)$ and in its Adriamycin-resistant counterpart H69AR $\left(\mathrm{LC}_{50}\right.$ of $4.5 \pm$ $0.9 \mu \mathrm{mol} / \mathrm{L}$; Fig. 1F).

NBDHEX Induces Necrosis in H69 Cells and a DoseDependent Apoptosis in the H69AR Cell Line

To characterize the type of cell death induced by NBDHEX, we evaluated the amount of apoptotic/necrotic cells in both H69AR and H69 cell lines after incubation with the drug. Figure 2 reports the percentages of cells undergoing necrosis
(Fig. 2A) or apoptosis (Fig. 2B and C) after incubation with NBDHEX. The occurrence of apoptosis in H69AR was revealed by the presence of cells with fragmented chromatin (data not shown). Time course analysis of cell death showed that apoptosis occurred within $3 \mathrm{~h}$ and continued to increase throughout the incubation period. Moreover, a clear dose-dependent effect was observed after $24 \mathrm{~h}$ of incubation with different amounts of NBDHEX. By contrast, the $\mathrm{H} 69$ cell line seemed quite insensitive to the treatment up to $\sim 1 \mu \mathrm{mol} / \mathrm{L}$ NBDHEX, whereas a further increase of drug concentration caused cell death by necrosis as determined by trypan blue staining. The necrotic H69 cells exhibited cytoplasmic swelling and diffuse nonfragmented

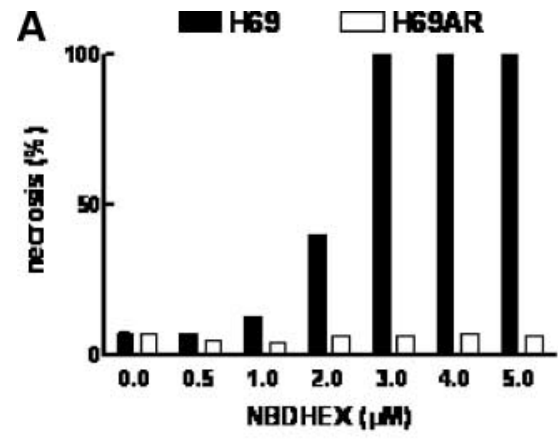

D

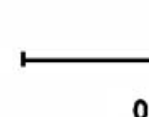

0
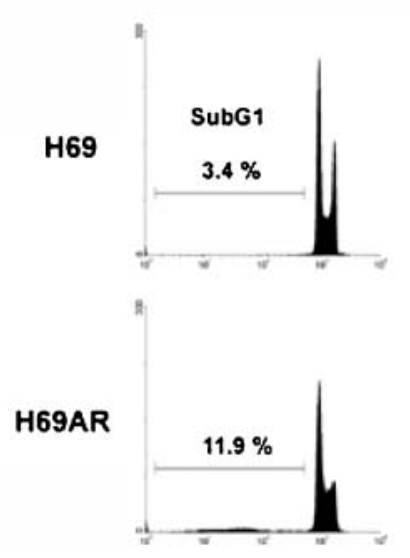

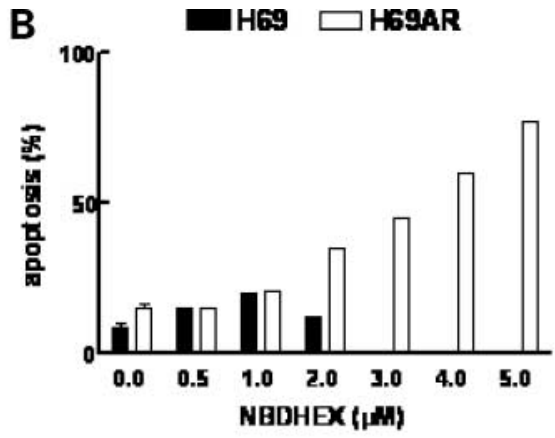

$\mu$ M NBDHEX

5
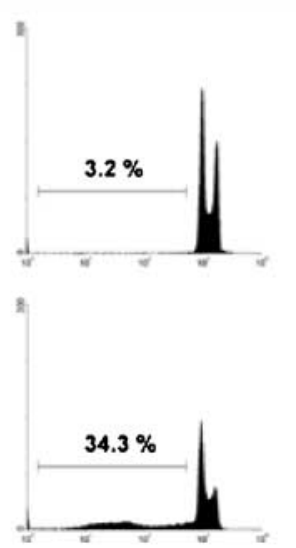

C

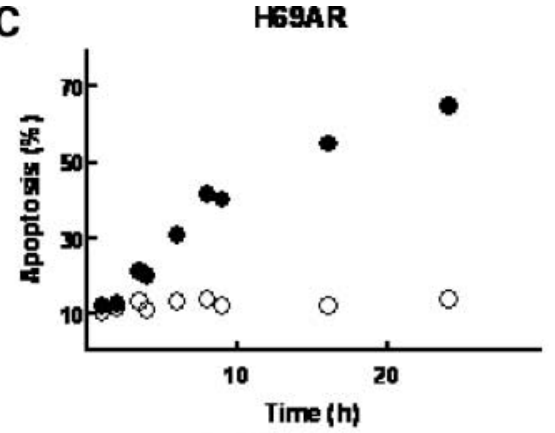

E

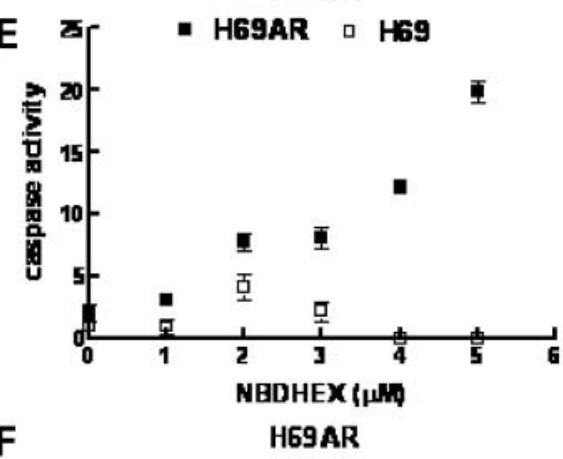

F

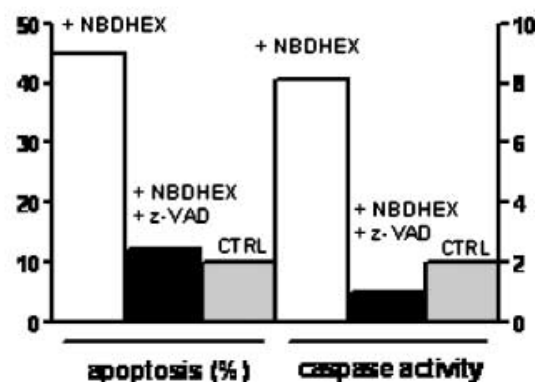

Figure 2. Cell death induction by NBDHEX. Cell death was determined in both $\mathrm{H} 69$ and H69AR cells after 24 h of incubation with several amounts of NBDHEX (from 0 to $5 \mu \mathrm{mol} / \mathrm{L}$ ). The histograms represent the frequency of necrotic (A) and apoptotic (B) SCLC cells as determined by trypan blue permeability and nuclear staining with Hoechst 33342, respectively. Results are expressed as a percentage of total cells and are representative of three independent experiments. C, time course of apoptosis triggered by $3 \mu \mathrm{mol} / \mathrm{L}$ NBDHEX in H69AR cells. $\bullet$, NBDHEX-treated cells; $O$, untreated control cells. D, flow cytometric analysis done on both $\mathrm{H} 69$ and H69AR cells incubated with different amounts of NBDHEX (from 0 to $10 \mu$ mol/L). After $6 \mathrm{~h}$ of treatment, cell distribution, according to their DNA content, was measured after cell permeabilization and propidium iodide staining. The fraction of cells with sub- $\mathrm{G}_{1}$ DNA content (apoptotic cells) was calculated using WinMDI version 2.8 software and is indicated in each profile. $\mathbf{E}$, increase of caspase-3 activity in $\mathrm{H} 69$ and H69AR cell lines after $24 \mathrm{~h}$ of treatment with several amounts of NBDHEX (from 0 to $5 \mu$ mol/L). Activity is expressed as fluorescence change measured within 1 min from the substrate addition, normalized per number of cells. Points, mean of triplicate independent determinations; bars, SD. F, caspase-3 activity and apoptosis were also determined, after $24 \mathrm{~h}$ of NBDHEX treatment, on H69AR cells preincubated with $40 \mu \mathrm{mol} / \mathrm{L}$ of caspase inhibitor Z-VAD$\mathrm{fm} k$ before the addition of $3 \mu \mathrm{mol} / \mathrm{L}$ NBDHEX and maintained throughout the experiment. Results are representative of three independent experiments that gave similar results. 
Figure 3. NBDHEX-induced apoptosis in H69AR cells is associated with the activation of $\mathrm{JNK} / \mathrm{c}-\mathrm{Jun}$ pathway. H69-sensitive and H69AR cells were treated with $3 \mu \mathrm{mol} / \mathrm{L}$ NBDHEX. The levels of JNK, C-Jun, and their phosphorylated isoforms were detected at indicated time points on total protein extracts $(20 \mu \mathrm{g})$ by Western blot analysis. $\alpha$-Tubulin was used as loading control. The immunoblot shown is from one experiment representative of three that gave similar results.

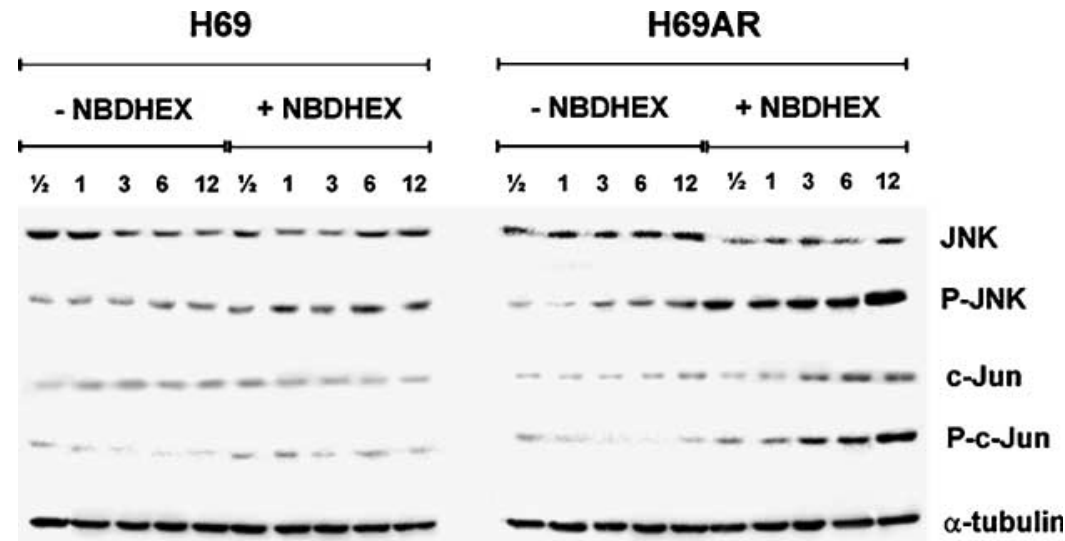

chromatin, suggesting that the phenomenon observed is primary necrosis instead of apoptosis-derived secondary necrosis (data not shown). To corroborate this hypothesis, we did cytofluorimetric analysis of both H69 and H69AR cells on staining with propidium iodide. H69AR treated with different amounts of NBDHEX showed a dosedependent increase of cell population with hypodiploid (sub-G $\mathrm{G}_{1}$ ) nuclei, in which DNA cleavage occurred as the ultimate step of apoptosis. Conversely, this trend was absent in the parental $\mathrm{H} 69$ cells, which showed no increase in sub-G $G_{1}$ peak when treated with the same amounts of NBDHEX (Fig. 2D). Taken together, these results indicate that the observed necrosis in H69 cell line is a bona fide apoptosis-independent, primary necrosis.

Activation of the caspase family proteases is also a central biochemical event occurring only in apoptotic cells. Therefore, the specific proteolytic activity of caspase-3 was assayed at intervals after drug treatment in both H69 and H69AR cell lines. Figure 2E shows caspase-3 activity after $24 \mathrm{~h}$ of incubation with different amounts of NBDHEX. H69 cells showed only a slight increase in caspase-3 activity after $2 \mu \mathrm{mol} / \mathrm{L}$ NBDHEX treatment, which quickly dropped to background levels by increasing drug concentration. In contrast, caspase- 3 activity was already observed in H69AR cells treated with $1 \mu \mathrm{mol} / \mathrm{L}$ NBDHEX and increased progressively with the increase of NBDHEX concentration. Caspase- 3 inactivation by the general caspase inhibitor Z-VAD-fmk fully prevented apoptosis in H69AR cells (Fig. 2F), so confirming the caspase involvement in the apoptotic response of cells exposed to NBDHEX.

NBDHEX-Induced Apoptosis Is Associated with the JNK/c-Jun Activation

Mitogen-activated protein kinases (MAPK) are important components of signaling pathways, which transduce extracellular stimuli into intracellular responses. It has been reported that NBDHEX is able to induce the activation of JNK pathway in a variety of cancer cells $(11,20)$. Therefore, the effect of NBDHEX on the phosphorylation/ activation of this MAPK was examined both in H69 cell line and in its Adriamycin-resistant counterpart. As shown in Fig. 3, treatment with NBDHEX increased the phosphory- lation of JNK in H69AR cells in a time-dependent fashion; phosphorylated JNK activation occurred as early as $30 \mathrm{~min}$ and remained at high levels for at least $12 \mathrm{~h}$. c-Jun, a direct downstream transcription factor activated by JNK, was also increased in the H69AR cells after $3 \mathrm{~h}$ of NBDHEX treatment, suggesting an involvement of the JNK/c-Jun pathway in the NBDHEX-induced apoptosis.

In contrast, neither change in the intensity of the immunoreactive band of phosphorylated JNK nor c-Jun activation was detected in the NBDHEX-treated H69 cells. It is well known that the GST isoenzyme GSTP1-1 inhibits JNK activity by forming a GSTP1-1-JNK heterocomplex (21). Therefore, to rule out the possibility that a higher GSTP1-1 expression in $\mathrm{H} 69$ cells could be responsible for the lack of JNK activation (11), the amount of this enzyme was determined both in the H69 cell line and in its MDR counterpart. No differences in the GSTP1-1 levels were observed between these cell lines (Fig. 4A). Neither were any significant changes in the GST activity observed between NBDHEX-treated and untreated control cells up to $6 \mathrm{~h}$ of incubation (Fig. 4B). These findings indicate that NBDHEX triggers a different cell death mechanism in H69 cells.

\section{Intracellular Redox Status and p38 ${ }^{\text {MAPK }}$ Activation}

To evaluate the effects induced by NBDHEX on proapoptotic members of MAPK family, we next analyzed the change in the phosphorylation/activation of $\mathrm{p} 38^{\mathrm{MAPK}}$. Immunoblot analysis of $\mathrm{H} 69$ cell line showed a marked increase in phosphorylation of $\mathrm{p} 38^{\mathrm{MAPK}}$, which occurred within $30 \mathrm{~min}$ of drug treatment, whereas NBDHEX induced only a negligible increase of $\mathrm{p} 38^{\mathrm{MAPK}}$ phosphorylation in the H69AR cell line (Fig. 5A). It has been suggested that $\mathrm{p} 38^{\mathrm{MAPK}}$ senses very low changes of the intracellular redox state (22). Thus, to show a possible change of this redox system after NBDHEX treatment, we measured the intracellular content of both reduced and disulfide form of glutathione, the most abundant low molecular weight antioxidant within the cell. Parental H69 cell line showed an average intracellular concentration of $\mathrm{GSH}$ of $\sim 60 \mathrm{nmol} / \mathrm{mg}$ protein, a value 3-fold higher than that of the Adriamycin-resistant counterpart $(\sim 20 \mathrm{nmol} / \mathrm{mg}$ protein; Fig. 5B). Moreover, a time-dependent increase of GSSG content was detected in the NBDHEX-treated H69 
cell line but not in the H69AR cells (Fig. 5C). This increase was not due to a reduced activity of the NADPHdependent glutathione disulfide reductase that continuously reduces GSSG back into GSH. In fact, H69 cells show a GSH reductase activity of $0.090 \mathrm{unit} / \mathrm{mg}(\mu \mathrm{mol} / \mathrm{min} / \mathrm{mg}$ a $\mathrm{pH}$ 7.6), which is comparable with the value found in the resistant H69AR cell line (0.080 unit/mg). It is known that the intracellular redox status depends on the relative amounts of the GSH and GSSG (22) and that the oxidation of a limited amount of GSH into GSSG can drastically change this ratio, so leading to activation of $\mathrm{p} 38^{\mathrm{MAPK}}$ and subsequent apoptosis. This was already reported for the K562 cell line treated with NBDHEX (20). However, the imbalance of the intracellular redox state and the activation of $\mathrm{p} 38^{\mathrm{MAPK}}$ found in $\mathrm{H} 69$ cells were not followed by the apoptotic cascade, and in this case, cells died by necrosis.

A
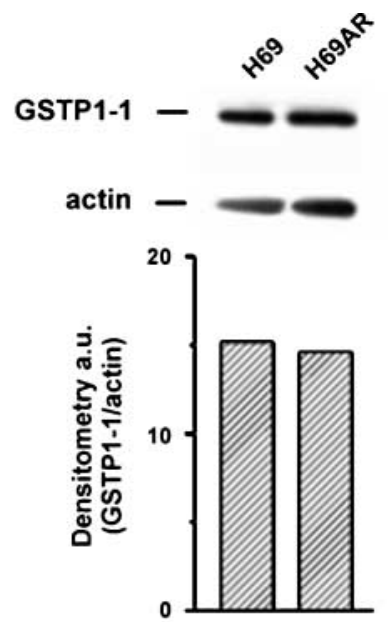

B

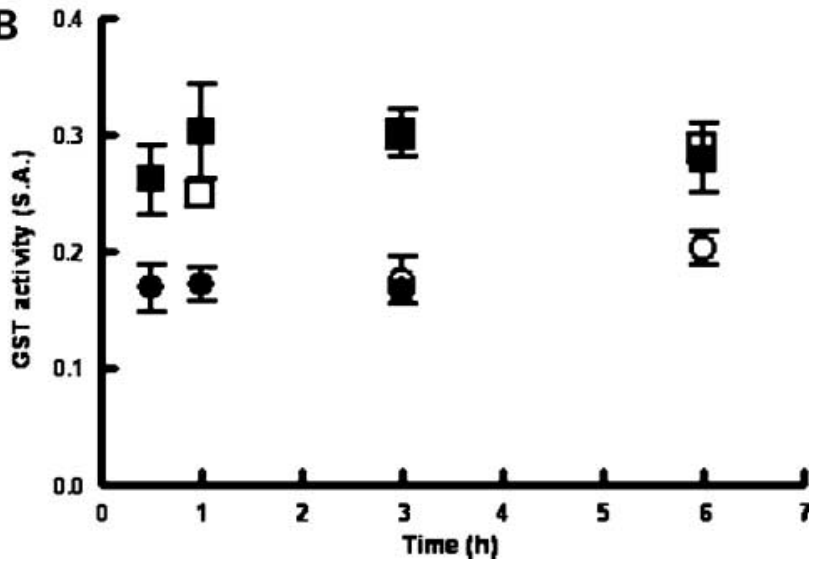

Figure 4. GST content in SCLC cell lines. A, $20 \mu \mathrm{g}$ of total protein extracts from H69 and H69AR cells were used for detection of GSTP1-1 protein content by Western blot. $\beta$-Actin was used as loading control. Immunoblot is from one experiment representative of three that gave similar results. Density of immunoreactive bands (reported below the immunoblot) was calculated using the software Quantity One, normalized for $\beta$-actin and reported as arbitrary units $(a . u$.). B, GST specific activity (S.A. $=\mu \mathrm{mol} \cdot \mathrm{min}^{-1} \cdot \mathrm{mg}^{-1}$ ) determined at indicated time points in $\mathrm{H} 69$ (๑) and H69AR ( $\mathbf{\square}$ ) cells after $3 \mu \mathrm{mol} / \mathrm{L}$ NBDHEX treatment; untreated control cells are also reported $(\mathrm{O}, \mathrm{H} 69 ; \mathrm{\square}, \mathrm{H} 69 \mathrm{AR})$. Points, mean of triplicate independent determinations; bars, SD.

\section{Effect of GSH Increase on Necrosis and Apoptosis Induced by NBDHEX}

An increase in intracellular oxidation may determine the selection between apoptosis and necrosis. In particular, suppression of apoptosis and the induction of necrosis is often associated with the intracellular GSH content $(23,24)$. Therefore, we tested the possibility of switching the NBDHEX-induced necrosis in apoptosis by increasing the intracellular level of GSH. We incubated H69 cells with glutathione ethylester, a permeant compound that directly increases the intracellular levels of GSH (25). Figure 5E shows that treatment with glutathione ethylester significantly prevented NBDHEX-mediated necrosis. In fact, the percentage of necrotic cells, after $24 \mathrm{~h}$ of treatment with $5 \mu \mathrm{mol} / \mathrm{L}$ NBDHEX, decreased from $100 \%$ to $36 \%$ in the presence of $1 \mathrm{mmol} / \mathrm{L}$ glutathione ethylester, although no switch from necrosis to apoptosis was observed. On the other hand, an increase of the intracellular levels of GSH partially improved $(\sim 25 \%)$ the survival of H69AR cells after $24 \mathrm{~h}$ of treatment with $5 \mu \mathrm{mol} / \mathrm{L}$ NBDHEX (Fig. 5D).

\section{Bcl-2 Content}

SCLCs, and particularly H69 cells, are known to express high levels of the antiapoptotic protein Bcl-2 (26). This was confirmed by Western blot analysis, which conversely showed a very low amount of Bcl-2 protein in the MPR1overexpressing H69AR cell line (Fig. 6). It can be hypothesized that the strong down-regulation of this antiapoptotic protein in H69AR cells may favor the occurrence of apoptosis following NBDHEX treatment.

\section{Discussion}

SCLC is characterized by a highly aggressive clinical course and a strong tendency to develop distant metastases. This makes chemotherapy more effective than surgical or radiation therapy. Consequently, we have investigated the efficacy of the nitrobenzoxadiazole-derivative NBDHEX on the human SCLC H69 cell line and on its MDR variant H69AR. Surprisingly, we found that NBDHEX was cytotoxic in both cell lines with $\mathrm{LC}_{50} \mathrm{~S}$ in the micromolar range. Interestingly, this drug triggers two different types of cell death: a caspase-dependent apoptotic pathway in the Adriamycin-resistant H69AR and a necrotic phenotype in the parental H69 cell line. To explain this result, some of the internal cell factors that counteract apoptosis in lung cancer have been analyzed. We found higher GSH levels and Bcl-2 overexpression in H69 cells compared with levels found in the Adriamycin-resistant H69AR cells characterized by an almost undetectable Bcl-2 content. Such evidence matches several studies showing a positive correlation between elevated Bcl-2 and GSH levels and suppression of apoptosis $(27,28)$. The important role that these antiapoptotic factors play in lung cancer progression is confirmed by the action mechanism of Adriamycin, which triggers apoptosis in H69 cells by down-regulation of Bcl-2 expression and depletion of GSH (29). However, under our experimental conditions, neither GSH nor Bcl-2 content decreased in $\mathrm{H} 69$ cells after treatment 
A

H69

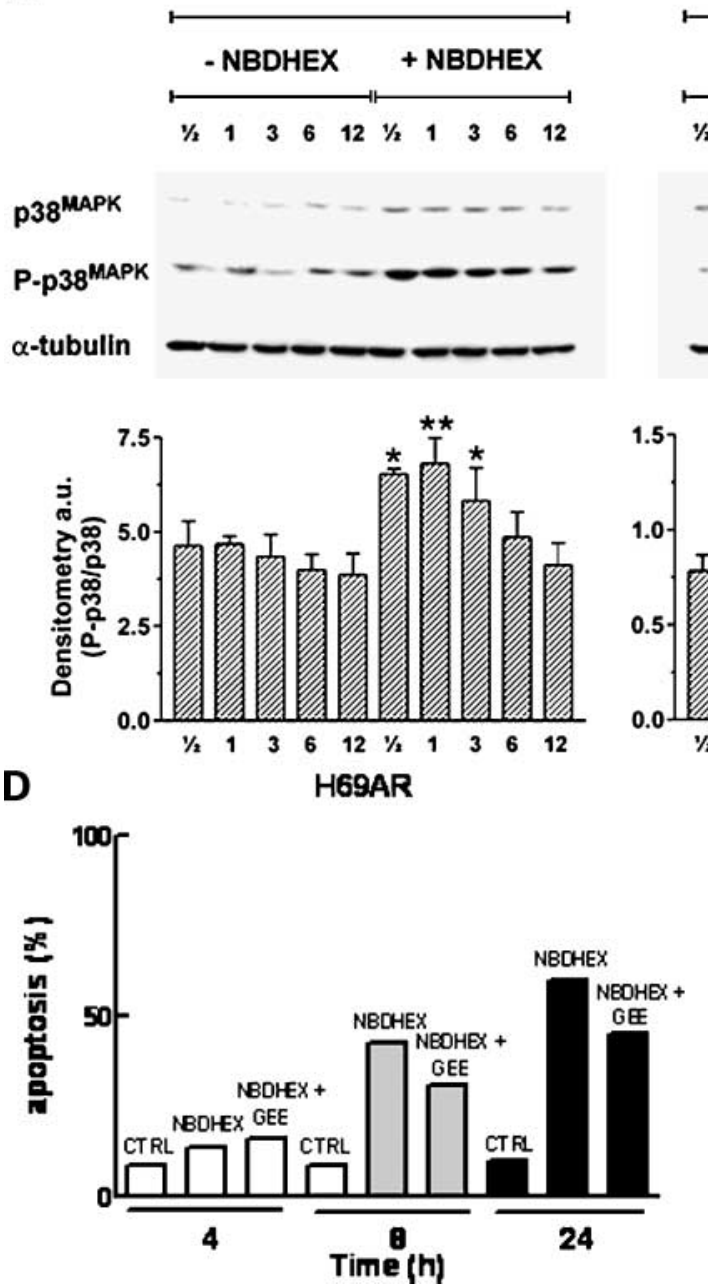

H69AR

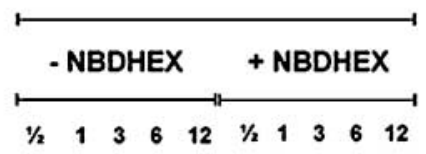

B

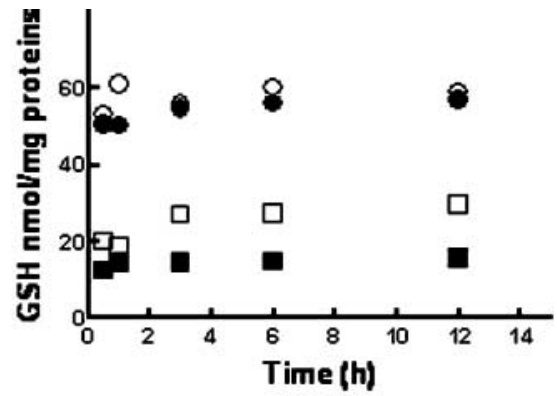

C

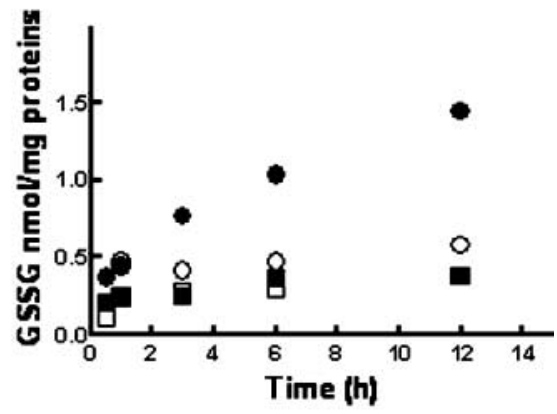

E

H69

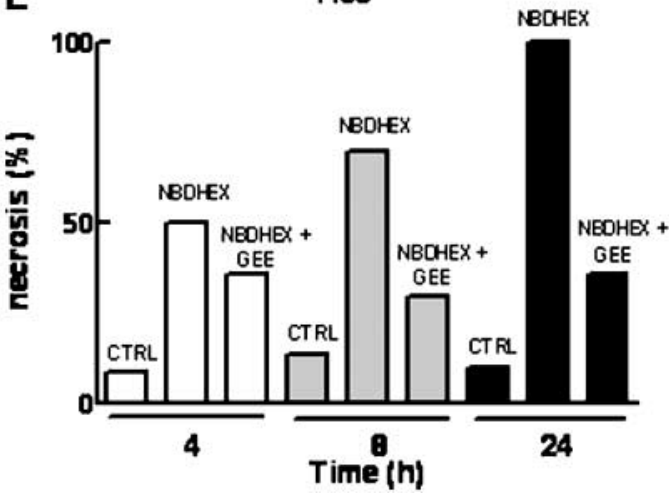

Figure 5. Redox status and p38 $8^{\mathrm{MAPK}}$ activation in SCLC cells. A, levels of p38 $8^{\mathrm{MAPK}}$ and its phosphorylated isoform were detected at indicated time points in both $\mathrm{H} 69$ and H69AR cells treated with $3 \mu \mathrm{mol} / \mathrm{L} \mathrm{NBDHEX.} \alpha$-Tubulin was used as loading control. The immunoblot shown is from one experiment representative of three that gave similar results. The density of immunoreactive bands (reported below the immunoblot) was calculated using the software Quantity One, normalized for $\alpha$-tubulin and reported as arbitrary units of the ratio between the phosphorylated and unphosphorylated form of p38 ( $P$-p38/p38). Starred columns, significant increase relative to the controls $(*, P<0.05 ; * * P<0.01)$. Intracellular GSH (B) and GSSG (C) levels were analyzed in $\mathrm{H} 69(\bullet)$ and H69AR ( $\boldsymbol{\square})$ cells at the indicated times after $3 \mu \mathrm{mol} / \mathrm{L}$ NBDHEX treatment. Untreated control cells were also analyzed $(O$, H69; $\square$, H69AR). Data are expressed as nmol of GSH or GSSG equivalents/mg total protein and are representative of three independent experiments that gave similar results. H69AR (D) and H69 (E) cell lines were preincubated with $1 \mathrm{mmol} / \mathrm{L}$ glutathione ethylester (GEE) for $1 \mathrm{~h}$ before the addition of $5 \mu$ mol/L NBDHEX. At the indicated times, the percentage of apoptosis in H69AR cells was determined by flow cytometric analysis after cell permeabilization and propidium iodide staining. The percentage of necrosis in $\mathrm{H} 69$ cells was determined after staining with trypan blue. Results are representative of three independent experiments that gave similar results.

with NBDHEX (Figs. 5 and 6, respectively). Moreover, an increase of intracellular GSH concentration did not switch necrosis to apoptosis, suggesting that the amount of intracellular GSH does not control by itself the mode of cell death in $\mathrm{H} 69$ cell line. It has also been reported that $\mathrm{Bcl}-2$ influences intracellular signaling cascades, thereby preventing apoptosis. In particular, Bcl-2 blocks the activation of the JNK signaling pathway by various apoptotic stresses (i.e., staurosporine UV light and anisomycin) by suppressing the stimulation of the upstream protein kinase MAPK kinase kinase $(30,31)$. These findings may explain the lack of JNK/c-Jun activation and the absence of apoptosis in H69 cells despite their being provided with an effective apoptotic machinery. The resulting necrosis observed after NBDHEX treatment may constitute a default cell death pathway induced when the activation of specific signal transduction cascades leading to cell death is inhibited (32). In spite of the adverse effects of necrosis, it could be therapeutically advantageous to trigger cell death by necrosis in lung cancer cells selected to resist apoptosis and characterized by extremely poor prognosis.

As far as MDR H69AR cells are concerned, we have found that the MRP1 transporter overexpressed in this cell line does not counteract the cytotoxicity of NBDHEX, 


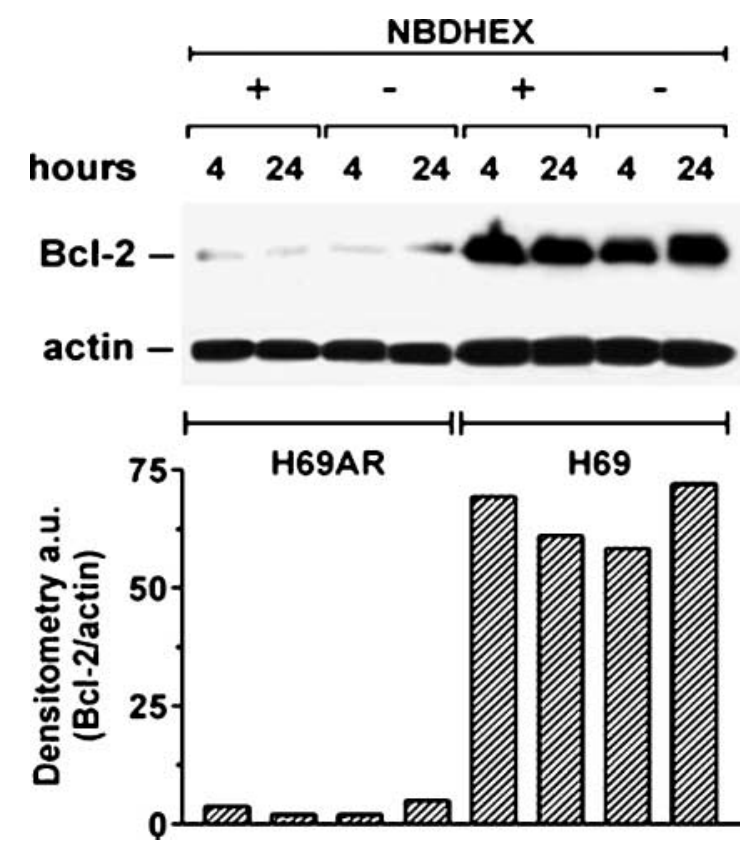

Figure 6. Bcl-2 expression in SCLC cells. The expression of Bcl-2 was examined in both $\mathrm{H} 69$ and H69AR cells by immunoblot analysis. At the indicated times, total protein extracts $(20 \mu \mathrm{g})$ from untreated and $3 \mu \mathrm{mol} / \mathrm{L}$ NBDHEX - treated cells were subjected to Western blot analysis. $\beta$-Actin was used as loading control. The density of immunoreactive bands (reported below the immunoblot) was calculated using the software Quantity One, normalized for $\beta$-actin and reported as arbitrary units.

which is not a substrate for this pump. NBDHEX induces a caspase-dependent apoptosis and this process may be promoted by the very low amount of Bcl-2 found in H69AR cells. The decrease of this antiapoptotic protein seems linked to the MDR phenotype. In fact, previous data show the concomitant down-regulation of $\mathrm{Bcl}-2$ gene in response to a significant overexpression of the MRP1 gene in H69 cells $(33,34)$. Moreover, it has been shown that the amount of intracellular GSH levels is a critical factor for MRP1expressing cells. In particular, MRP1 expression causes a reduction in cellular GSH levels and this makes H69AR cells hypersensitive to drugs, such as verapamil and flavonoids, which increase the MRP1-mediated GSH transport. Accordingly, addition of GSH to the medium efficiently prevents apoptosis induced by these drugs in MRP1-expressing cells $(35,36)$. Under our experimental conditions, we observed that an increase in intracellular GSH levels partially improved the survival of H69AR cells treated with NBDHEX. Moreover, NBDHEX does not seem to affect the correct redox equilibrium of this cell line. In fact, no decrease in GSH levels and no accumulation of oxidized glutathione are observed during NBDHEX treatment (Fig. 5). We cannot rule out the hypothesis that GSH could be extruded from the cells under our experimental conditions; however, this probably occurs in a long time frame so as to allow the cell to induce GSH neosynthesis. Therefore, the role played by GSH in the apoptosis of NBDHEX-treated H69AR cells is not completely under- stood at this time and will be the goal of future investigations. Whatever the case, a down-regulation of factors crucial for cell survival makes H69AR cells more sensitive to the cytotoxic action of drugs that are not MRP1 substrates. Actually, MRP1 transports not only a wide variety of neutral hydrophobic compounds but also numerous glutathione, glucuronate, and sulfate conjugates (13), and only a few anticancer drugs are poor substrates (37) or not substrates of MRP1 at all (38). Thus, many attempts have been made to identify agents able to reverse the MDR mediated by MRP1 (39). However, many of these molecules in clinical trials revealed intrinsic toxicity at doses effective in reversing MDR (40). Finally, to enhance the efficacy of chemotherapy in MDR lung cancer, complex strategies based on the simultaneous administration of an anticancer drug and of antisense oligonucleotides targeting antiapoptotic defense have been proposed (41). Taken together, all this evidence emphasizes the potential anticancer role of NBDHEX, a compound that is effective against drug-resistant tumors overexpressing both P-gp (11) and MRP1 and that also shows low toxicity in vivo (20).

\section{References}

1. Ginsberg RJ, Vokes EE, Raben A. Non-small-cell lung cancer. In: de Vita VT, Hellmann S, Jr., Rosenberg SA, editors. Cancer: principles and practice of oncology. Philadelphia (PA): Lippincott-Raven; 1997. p. $858-910$

2. Ihde DC, Pass HI, Glattstein EJ. Small-cell lung cancer. In: de Vita VT, Hellmann S, Jr., Rosenberg SA, editors. Cancer: principles and practice of oncology. Philadelphia (PA): Lippincott-Raven; 1997. p. $911-49$.

3. Fisher DE. Apoptosis in cancer therapy: crossing the threshold. Cell $1994 ; 78: 539-42$.

4. Thompson CB. Apoptosis in the pathogenesis and treatment of disease. Science 1995;267:1456-62.

5. Zangemeister-Wittke U, Schenker T, Luedke GH, Stahel RA. Synergistic cytotoxicity of $\mathrm{bcl}-2$ anti-sense oligodeoxynucleotides and etoposide, doxorubicin and cisplatin on small-cell-lung-cancer cell lines. $\mathrm{Br} \mathrm{J}$ Cancer 1998;78:1035-42.

6. Ziegler A, Luedke GH, Fabbro D, Altmann $\mathrm{KH}$, Zangemeister-Wittke $\mathrm{U}$. Induction of apoptosis in small-cell-lung-cancer cells by an anti-sense oligodeoxynucleotide targeting the bcl-2 coding sequence. J Natl Cancer Inst 1997;89:1027-36.

7. Haggins CF. ABC transporters: from microorganisms to man. Annu Rev Cell Biol 1992;8:67-113.

8. Gottesman MM, Pastan I, Ambudrak SV. P-glycoprotein and multidrug resistance. Curr Opin Genet Dev 1996;6:610 - 7.

9. Cole SP, Deeley RG. Multidrug resistance mediated by the ATP-binding cassette transporter protein MRP. Bioessays 1998;20:931-40.

10. Mirsk SEL, Gerlach JH, Cole SPC. Multidrug resistance in a human small cell lung cancer cell line selected in adriamycin. Cancer Res 1987; 47:2594-8.

11. Turella $P$, Filomeni $G$, Dupuis $M L$, et al. A strong glutathione $S$-transferase inhibitor overcomes the P-glycoprotein-mediated resistance in tumor cells. 6-(7-Nitro-2,1,3-benzoxadiazol-4-ylthio)hexanol (NBDHEX) triggers a caspase-dependent apoptosis in MDR1-expressing leukemia cells. J Biol Chem 2006;281:23725-32.

12. Ricci G, De Maria F, Antonini G, et al. 7-Nitro-2,1,3-benzoxadiazole derivatives, a new class of suicide inhibitors for glutathione $S$-transferases. Mechanism of action of potential anticancer drugs. J Biol Chem 2005;280:26397-405.

13. Bakos É, Homolya L. Portrait of multifaceted transporter, the multidrug resistance-associated protein 1 (MRP1/ABCC1). Pflugers Arch 2007;453:621 - 41

14. Kruh GD, Belinsky MG. The MRP family of drug efflux pumps. Oncogene 2003;22:7537-52. 
15. Hipfner DR, Gao M, Scheffer G, Scheper RJ, Deeley RG, Cole SP Epitope mapping of monoclonal antibodies specific for the 190-kDa multidrug resistance protein (MRP). Br J Cancer 1998;78:1134-40.

16. Cianfriglia M, Willingham MC, Tombesi M, Scagliotti GV, Frasca G, Chersi A. P-glycoprotein epitope mapping. I. Identification of a linear human-specific epitope in the fourth loop of the P-glycoprotein extracellular domain by MM4.17 murine monoclonal antibody to human multidrug resistant cells. Int J Cancer 1994;56:153-60.

17. Skehan $P$, Storeng $R$, Scudiero $D$, et al. New colorimetric cytotoxicity assay for anticancer-drug screening. J Natl Cancer Inst 1990;82 $1107-12$.

18. Gekeler V, Ise W, Sanders KH, Ulrich WR, Beck J. The leukotriene LTD4 receptor antagonist MK571 specifically modulates MRP associated multidrug resistance. Biochem Biophys Res Commun 1995;208:345 - 52

19. Reed DJ, Babson JR, Beatty PW, Brodie AE, Ellis WW, Potter DW. High-performance liquid chromatography analysis of nanomole levels of glutathione, glutathione disulfide, and related thiols and disulfides. Anal Biochem 1980; 106:55 - 62.

20. Turella $P$, Cerella C, Filomeni G, et al. Proapoptotic activity of new glutathione $S$-transferase inhibitors. Cancer Res 2005;65:3751-61.

21. Townsend DM, Tew KD. The role of glutathione- $S$-transferase in anticancer drug resistance. Oncogene 2003;22:7369- 75.

22. Filomeni G, Rotilio G, Ciriolo MR. Glutathione disulfide induces apoptosis in U937 cells by a redox-mediated p38 MAP kinase pathway. FASEB J 2003;17:64 - 6

23. Vairetti $M$, Ferrigno $A$, Bertone $R$, Richelmi $P$, Bertè $F$, Freitas Apoptosis vs. necrosis: glutathione-mediated cell death during rewarming of rat hepatocytes. Biochim Biophys Acta 2005;1740:367 - 74.

24. Sancho $P$, Fernàndez $C$, Yuste $V J$, et al. Regulation of apoptosis/ necrosis execution in cadmium-treated human promonocytic cells under different forms of oxidative stress. Apoptosis 2006;11:673-86.

25. Martensson J, Han J, Griffith OW, Meister A. Glutathione ester delays the onset of scurvy in ascorbate-deficient guinea pigs. Proc Natl Acad Sci U S A 1993;90:317-21.

26. Leech SH, Olie RA, Gautschi O, et al. Induction of apoptosis in lungcancer cells following bcl-xL anti-sense treatment. Int J Cancer 2000;86: $570-6$.

27. Mirkovic N, Voehringer DW, Story MD, McConkey DJ, McDonnell TJ Meyn RE. Resistance to radiation-induced apoptosis in Bcl-2-expressing cells is reversed by depleting cellular thiols. Oncogene 1997;15: $1461-70$

28. Voehringer DW, Meyn RE. Redox aspects of Bcl-2 function. Antioxid Redox Signal 2000;2:537-50
29. Sharma A, Patrick B, Li J, et al. Glutathione S-transferases as antioxidant enzymes: small cell lung cancer (H69) cells transfected with hGSTA1 resist doxorubicin-induced apoptosis. Arch Biochem Biophys 2006;452:165 - 73

30. Park DS, Stefanis L, Yan CY, Farinelli SE, Greene LA. Ordering the cell death pathway. Differential effects of BCL2, an interleukin-1-converting enzyme family protease inhibitor, and other survival agents on JNK activation in serum/nerve growth factor-deprived PC12 cells. J Biol Chem 1996;271:21898-905

31. Park J, Kim I, Oh YJ, Lee K, Han PL, Choi EJ. Activation of c-Jun $\mathrm{N}$-terminal kinase antagonizes an anti-apoptotic action of Bcl-2. J Bio Chem 1997:272:16725-8.

32. Golstein $P$, Kroemer G. Cell death by necrosis: towards a molecular definition. Trends Biochem Sci 2007:32:37-43.

33. Van Hille B, Lohri A, Reuter J, Herrmann R. Assessment of druginduced dysregulations among seven resistance-associated genes in human tumour cell lines. Anticancer Res 1996;16:3531 - 6.

34. Henness S, Davey MW, Harvie RM, Davey RA. Fractionated irradiation of $\mathrm{H} 69$ small-cell lung cancer cells causes stable radiation and drug resistance with increased MRP1, MRP2, and topoisomerase Ilo expression. Int J Radiat Oncol Biol Phys 2002;54:895-902.

35. Trompier D, Chang X-B, Barattin R, et al. Verapamil and its derivative trigger apoptosis through glutathione extrusion by multidrug resistance protein MRP1. Cancer Res 2004;64:4950-6.

36. Laberge R-M, Karwatsky J, Lincoln MC, et al. Modulation of GSH levels in ABCC1 expressing tumor cells triggers apoptosis through oxidative stress. Biochem Pharmacol 2007;73:1727-37.

37. Rózalski M, Krajewska U, Panczyk M, et al. Synthesis and biological evaluation of 4-methylideneisoxazolidin-5-ones - a new class of highly cytotoxic $\alpha$-methylidene- $\gamma$-lactones. Eur J Med Chem 2007;42:248 - 55.

38. Efferth T. Mechanistic perspectives for 1,2,4-trioxanes in anti-cancer therapy. Drug Resist Updat 2005;8:85-97.

39. Boumendjel A, Baubichon-Cortay $H$, Trompier D, Perrotton $T$, Di Pietro A. Anticancer multidrug resistance mediated by MRP1: recent advances in the discovery of reversal agents. Med Res Rev 2005;25: $453-72$

40. Tan B, Piwnica-Worms D, Ratner L. Multidrug resistance transporters and modulation. Curr Opin Oncol 2000;12:450-8

41. Pakunlu RI, Wang $Y$, Tsao $W$, Pozharov $V$, Cook TJ, Minko $T$. Enhancement of the efficacy of chemotherapy for lung cancer by simultaneous suppression of multidrug resistance and antiapoptotic cellular defense: novel multicomponent delivery system. Cancer Res 2004;64:6214-24 